\title{
Effect of four sessions of aerobic exercise with abdominal radiofrequency in adipose tissue in healthy women: Randomized control trial
}

\author{
Ana Luísa Vale PT $^{1}$ | Ana Sofia Pereira PT ${ }^{1}$ | Andreia Morais PT $^{1}$ | Paulo de Carvalho PhD ${ }^{1}$ \\ Rui Vilarinho $\mathrm{MSc}^{1}$ | Adriana Mendonça PhD ${ }^{2}$ | Andreia Noites PhD ${ }^{1,3}$ \\ ${ }^{1}$ Department of Physiotherapy, School \\ of Health, Polytechnic Institute of Porto, \\ Portugal \\ ${ }^{2}$ Department of Applied Physiotherapy, \\ Health Sciences Institute, Federal University \\ of Triângulo Mineiro, Uberaba, Brazil \\ ${ }^{3}$ Center for Rehabilitation Research \\ (CIR)- Human Movement System (Re) \\ habilitation, Polytechnic Institute of Porto, \\ Portugal
}

\begin{abstract}
Background: Abdominal adiposity is a cardiovascular risk factor; it is assumed that the combination of radiofrequency and physical exercise may decrease this excess of adipose tissue.

Purpose: To analyze the effect of four intervention sessions on abdominal adipose tissue.

Methodology: The study analyzes the effect of four sessions of aerobic exercise combined with radiofrequency. Participants were 28 healthy female volunteers, aged 18-28 years, randomly assigned to an experimental group and a placebo group. They were characterized by a sociodemographic questionnaire, the International Physical Activity Questionnaire and the Food Frequency Questionnaire. The groups performed four sessions of abdominal radiofrequency followed by moderate aerobic exercise, assessing body mass, body mass index, fat mass, waist circumference, waist/height ratio, subcutaneous adipose thickness, and horizontal abdominal adipose fold at the first and fourth sessions. In the experimental group, a percentage of potency was used that allowed to maintain the temperature between $40^{\circ} \mathrm{C}$ and $42^{\circ} \mathrm{C}$ to the epidermis. In the placebo group, the power of the radiofrequency was $0 \mathrm{~W}$. To compare results, Student's $t$ test was used for a significance level of 0.05 .
\end{abstract}

Results: There was a statistically significant decrease in waist circumference, abdominal subcutaneous adipose thickness, and horizontal abdominal fold in the experimental group compared to the placebo group $(P<$ 0.05).

Conclusion: Combined exercise intervention with radiofrequency has been shown to be effective in reducing abdominal adiposity. 


\section{1 | INTRODUCTION}

Obesity is currently a worldwide epidemic, with the World Health Organization (WHO) estimating that at least 2.8 million people die every year as a result of overweight and obesity. ${ }^{1}$

Changes in dietary habits associated with sedentarism have contributed to the increase in the prevalence of obesity in Portugal in the last 40 years, with overweight already reaching more than half of the Portuguese adult population. ${ }^{2}$

The evidence shows that accumulation of adipose tissue in the abdominal region is a risk factor for dyslipidemias, hypertension, insulin resistance, among others. ${ }^{3}$

Physical exercise is a very important agent in the fight against overweight and, depending on the modality, intensity, and duration of exercise, the energy substrate used may be another type of source other than lipids. ${ }^{4,5}$

Aerobic exercise is a modality capable of decreasing body fat, and it can increase fatty acid oxidation 5-10 times in relation to the resting state. ${ }^{5}$ The evidence suggests that the prescription of exercise to decrease adipose mass should focus on a high training volume (30-60 minutes) with moderate intensity (40\%-60\% of the Heart Rate of Reservation), performed regularly and mobilizing large muscle groups. ${ }^{4,5}$

However, during exercise, the consumption of energy reserves appears to be global. ${ }^{4,5}$

In order to look for strategies that act on abdominal adiposity, radiofrequency (RF) can be considered a complementary strategy. This electrotherapy approach is a form of high-frequency electromagnetic energy that efficiently heats the adipose tissue, which allows elevating local cell metabolism by increasing lipolysis. That is, RF promotes the reversible biochemical phenomenon, where triglyceride release and breakdown occurs in lipid droplets of adipocytes. This process culminates with the generation of glycerol and nonesterified fatty acids that can be used for energy production. This release of lipid content leads to the hypotrophy of adipocytes, that is, a decrease in their volume. ${ }^{6}$

If mobilized lipids are not consumed, they will be converted to triglycerides and stored again in adipose tissue or ectopically accumulated in the liver, overloading this organ and leading to the production of low-density (LDL) cholesterol. ${ }^{7}$ Thus, a combined approach with exercise associated with RF can make intervention more efficient for lipid removal.

Although there are studies that point to the possibility of increased lipolytic activity and adipocyte hypotrophy after RF application, ${ }^{8}$ we found no articles correlating RF with exercise, since none of these studies present experimental and placebo division, ${ }^{6,9}$ which corroborates the relevance of this study.

The main objective of this study was to analyze the effect of four intervention sessions on abdominal fat, specifically on body mass, body mass index (BMI), total fat mass, waist circumference, waist/ height ratio, subcutaneous fat thickness, and abdominal horizontal fold.

\section{2 | METHODS}

\section{1 | Study design}

This study was classified as a randomized controlled parallel and double blind, taking into account the criteria of CONSORT. ${ }^{10}$

Participants were recruited from an initial assessment of body composition and anthropometry, performed at the Allied School of Health in Porto in May, in which seventy-five students participated.

The intervention protocol, applied in June in the same facilities, consisted of four sessions where, in each one, the participants allocated to the experimental group (EG) were subjected to an abdominal $\mathrm{RF}$ protocol (with percentage of power to maintain the temperature from $40^{\circ} \mathrm{C}$ to $42^{\circ} \mathrm{C}$ to the epidermis), followed by an aerobic physical exercise protocol. The placebo group (PG) performed the RF protocol with power of $\mathrm{O} \mathrm{W}$, followed by the protocol of aerobic physical exercise similar to that of GE.

Anthropometric measures and body composition (body mass, Body Mass Index, total fat mass, waist circumference, waist/height ratio, horizontal abdominal fold) and subcutaneous fat thickness were assessed using ultrasound before intervention.

In the last session, anthropometric measures, body composition, and ultrasonographic evaluation were repeated.

The four intervention sessions were conducted with a minimum interval of 24 hours and a maximum of 72 hours.

\subsection{Sample selection and inclusion criteria}

The target population was the female students of the Allied School of Health in Porto.

The G-Power 3.0.10 (Universität Düsseldorf) software was used to calculate the number of participants required in each group (EG and PG), for a power of $80 \%$. This calculation was based on a similar article, in which the anthropometric measurements and the thickness of the subcutaneous adipose tissue were also evaluated by ultrasonography. ${ }^{11} \mathrm{~A}$ minimum of 17 participants in each group was determined as necessary, based on the ultrasonography values of the referred study.

All students of the Allied School of Health, in the scope of the present study, were invited through posters, social networks, class emails, and institutional emails to participate in an initial assessment of body composition. In the course of this evaluation, individuals who met the eligibility criteria were invited orally to join this study, after a brief explanation. This evaluation consisted in the application of a sociodemographic questionnaire, in the register of anthropometric measurements and body composition (height, body mass, Body Mass Index (BMI), total fat mass, waist circumference, horizontal abdominal fold), and resting heart rate.

Each participant was assigned a numerical code to proceed to random allocation in each group.

Inclusion criteria were defined as individuals between 18 and 35 years of age with a BMI between $18.5 \mathrm{~kg} / \mathrm{m}^{2}$ (normal) and $29.9 \mathrm{~kg} / \mathrm{m}^{2}$ (overweight). 
TABLE 1 Intraobserver reliability values obtained in the pilot study

\begin{tabular}{ll} 
& Intraclass correlation (ICC) \\
\hline Waist circumference $(\mathrm{cm})$ & 0.951 \\
\hline Horizontal abdominal fold $(\mathrm{mm})$ & 0.970 \\
\hline Subcutaneous fat thickness (ultrasonography; $\mathrm{mm})$ & 0.996 \\
\hline
\end{tabular}

Exclusion criteria included smoking and/or alcohol habits; dietary restrictions or involvement in hypo or hypercaloric diet; involvement in programs of treatment of cellulite or weight loss, with application of creams and other therapeutic procedures; pregnant or postpartum $<1$ year; holders of electronic or metal devices (pacemaker, intrauterine device); metabolic dysfunctions (including dyslipidemias), cardiovascular, respiratory, hematological, renal, dermatological or digestive disorders; history of oncological pathology; musculoskeletal injury that interferes with the practice of cycle ergometer; sensitivity changes; cognitive deficit. ${ }^{12,13}$

The distribution of the participants by groups was made by placing a paper with the code of each participant in a black bag. An external element of the project removed from the bag the papers with the codes being that it was defined that the first 18 consecutive papers corresponded to the codes of the participants allocated to the EG and the remaining 17 would be the participants allocated to the PG. Neither participants nor the evaluators were aware of the form of allocation so that double blindness could be established in the study. The only investigator who had access to the allocation of the participants was the one who applied the RF protocol.

\section{3 | Instruments}

\subsection{1 | Questionnaires}

A questionnaire was developed to collect sociodemographic data and to verify compliance with the necessary criteria for participation in the study (https://docs.google.com/forms/d/1COKkJVbOW tkbvOBROoVj6FUp8n7C1vhri5pO4FOHISk/edit?usp=drive_web; Annex 2).

The short version of the International Physical Activity Questionnaire (IPAQ) was used to determine the participants' previous level of physical activity. This allows the assessment of the duration, type, frequency, and intensity of physical activity performed by the participant in the last 7 days. ${ }^{14}$ It is validated for the Portuguese population by Craig et al, ${ }^{15}$ with a concurrent validity of 0.49 , reliability with Spearman's $\sigma$ of 0.77 and reproducibility coefficient of 0.83 .

The Food Frequency Questionnaire (FFQ) was used to measure the food intake of the participants in the last 12 months. This questionnaire is of a quick and easy application, allowing to identify consumption patterns of the participants. ${ }^{16}$ The QFA is validated for the Portuguese population with mean values of correlations with daily food registers of 0.54 . The reproducibility of the questionnaire presented an average correlation value of 0.57 for the 22 nutrients. ${ }^{16}$

\subsection{2 | Anthropometric measurements}

In order to measure BMI (BMI $=$ Weight $/$ Height ${ }^{2}\left[\mathrm{~kg} / \mathrm{m}^{2}\right]$; General Health Department, 2013) and the percentage of total fat mass, the Tanita (model BC-545N Inner Scan TM; Amsterdam, Netherlands) was used. This instrument has a maximum capacity of $150 \mathrm{~kg}$ and a precision of $0.1 \mathrm{~kg}$ per $\mathrm{kg}$. It presents a correlation coefficient with dual energy radiological absorptiometry (DEXA) between 0.88 and $0.89 .^{17}$

To measure the height and waist circumference of the participants, a tape measure of the brand COMED $^{\circledR}$ was used. This inelastic and flexible tape presents excellent correlation with magnetic resonance, ${ }^{18}$ with an accuracy of $0.5 \mathrm{~cm}$ and a reproducibility coefficient of 0.91 . $^{19}$

The Harpen adipometer was used to estimate the amount of abdominal adiposity through the horizontal abdominal fold. It presents a precision of $99 \%$ and a correlation coefficient with DEXA of $0.93{ }^{17}$

\subsection{3 | Ultrasonography}

To measure the thickness of subcutaneous adipose tissue, the Viamo $^{\mathrm{TM}}$ ultrasound model, by Toshiba Medical Systems Corporation, was used with a $7.5 \mathrm{~Hz}$ wave frequency probe. It is an easy to apply, low-cost method that does not $(R=0.67){ }^{20}$

\section{4 | Procedures}

\subsection{1 | Pilot study}

A pilot study $(n=6)$ was carried out, which included the application of the sociodemographic questionnaire and the intervention procedure.

From the pilot study of the sociodemographic questionnaire, two questions were reformulated at the level of hyperlinks.

To test the exercise intervention protocol associated with RF, a female subject with characteristics similar to the sample participated. This subject fulfilled all the criteria and did not participate in the study.

In order to assess the intraobserver reliability of the anthropometric measures and the ultrasonography, a sample of six individuals with characteristics similar to those of the participants was used, but did not integrate this study. The retest was performed after 72 hours (a sufficiently short interval to allow no changes in the subjects, but long enough not to allow the evaluator to memorize). ${ }^{21}$ The reliability values given in Table 1 were obtained. 


\subsection{2 | Questionnaires application}

The sociodemographic questionnaire was completed during the initial evaluation.

The IPAQ and the QFA were filled in a computer provided by the researchers in the first session.

The IPAQ allowed calculating the MET-min/wk, according to the guidelines. $^{22}$

The results obtained in the QFA were processed through the Food Processor Plus program. ${ }^{16}$

\subsection{3 | Anthropometric measures, body composition, and ultrasonography}

For all measurements three measurements were taken and the mean of them was calculated.

To measure the height, the measurement tape was attached to the wall with tape. The participant was placed barefoot, with heels, buttocks, shoulder blades, and occipital next to the wall, keeping the feet to the width of the hips. Measurements were performed on expiratory apnea at tidal volume. ${ }^{4}$

The waist circumference was assessed in an orthostatic position, with upper limbs along the body, looking forward, with the tape placed parallel to the floor above the navel. Measurements were performed at the time of expiratory apnea at tidal volume. ${ }^{4}$

To obtain the body mass, fat mass percentage, and BMI, participants remained in the orthostatic position with bare feet on the scales sensors, grasping the hand electrodes parallel to the floor looking forward, with upper limbs along of the body, ${ }^{17}$ always guaranteeing the conditions determined by Tanita for its evaluation.

Adipometry was focused on the measurement of the horizontal abdominal fold. Measurements were performed $2 \mathrm{~cm}$ from the navel in the right hemitruncus, with the participant in an orthostatic position with feet to the width of the hips, upper limbs hanging along the body, and looking ahead. ${ }^{23}$ The adipose tissue was highlighted with the thumb and forefinger of the left hand, while the right hand held the adipometer Measurement was recorded after 3 seconds (for needle stabilization). ${ }^{23}$

Ultrasonography was used to determine the thickness of abdominal subcutaneous adipose tissue. This was done at the beginning of the study and at the end of the fourth session. Each participant assumed the position of dorsal decubitus with knees bent over in a marquise. Measurements were made $2 \mathrm{~cm}$ away from the umbilicus, in the right hemitruncus, with the probe placed perpendicular to the surface of the skin and oriented parallel to the alba line. The subcutaneous thickness was determined directly by the frozen images on the screen, at the end of the expiratory phase in apnea. ${ }^{24}$

\subsubsection{Determination of the Reserve Heart Rate (RHR) and Training Heart Rate (THR)}

For each participant, the theoretical maximum heart rate (HR max) was determined based on the Tanaka equation (FC $\max =208-0.7 \times$ Age $)^{4}$
Based on these values, using the Karvonen equation, the THR (T $\mathrm{HR}=$ HRrest + Intensity $\times \mathrm{RHR}$ ) the intensity of training was calculated for each participant, considering an intensity of $45 \%-55 \%$ of the RHR (RHR = HR max - HRrest $)^{4}$.

The determination of the resting HR was made after sitting 5-10 minutes, using the cardiofrequencymeter (for heart rate stabilization). ${ }^{25}$

\subsection{5 | RF protocol}

For the RF protocol, the BTL-6000 TR-Therapy apparatus (BTL Industry Ltd.) was used, with the 5-cm capacitive electrode.

Temperature monitoring was performed using a combination of two instruments. The Schliephak allows qualitative monitoring of temperature perception by the participant. It is included in the user's manual, and its use is indicated in conjunction with the quantitative temperature monitoring method. This scale consists of four levels (I-without perceiving heat, very low intensity, II-perception of moderate heat, low intensity, III-evident heat perception, moderate intensity, IV-Strong, but not unpleasant, intensity perception strong). ${ }^{26}$

Objective temperature monitoring was done using the FLIR E6 (Boston) thermograph. It has a frequency of $9 \mathrm{~Hz}$ and a margin of error of $<0.06^{\circ} \mathrm{C}^{27}$

The RF application was performed with the participants in the position of dorsal decubitus, with a cushion under the head and with the knees in flexion.

The RF was applied in the capacitive mode, with a power of $150 \mathrm{~W}$ (the percentage of power being adjusted according to the temperature, evaluated at each moment), and several slow passages were performed in the abdominal region (defining a square joining the anterior superior iliac spine and the last rib bilaterally). The cream supplied with the appliance (FIAB-Reference G016), a water-based cream for better heat conduction ${ }^{26}$ was used as the contact medium.

One-and-a-half minutes per applicator head area $(5 \mathrm{~cm}$ diameter electrode) was defined as the application time, aiming to reach and maintain a skin temperature of $40-42^{\circ} \mathrm{C}$, monitored with the thermograph and Schliephak Scale (comfortable warmth perception). ${ }^{26}$

The sessions were conducted with a minimum interval of 24 hours and a maximum of 72 hours.

\subsection{6 | Exercise protocol}

For the application of the physical exercise protocol, the HRrest, the THR interval, and the heart rate during training were monitored.

For that, the Polar FT7 cardiofrequencymeter was used with an accuracy of \pm 1 beats per minute (bpm). ${ }^{28}$ It is as reliable as the electrocardiogram to obtain heart rate (correlation of 0.981-0.998). ${ }^{29}$

The intensity monitoring was also done subjectively through the Subjective Perception Scale of Effort-Borg Scale. This scale ranges from 6 to 20, with 6 being extremely light exercise and 20 being maximum effort. ${ }^{30}$ It has very high correlation values with either heart rate or maximum $\mathrm{VO}_{2}$ values in healthy subjects $(0.80-0.90) .^{30}$ 
The aerobic exercise protocol was performed with the Monark cycle ergometer, shortly after the end of the RF protocol.

Before starting, the cardiofrequencymeter was placed on the participant and the saddle adjusted. The foot was centered on the pedal and, when the pedal was in the lower position, the knee was in slight flexion $\left(5-10^{\circ}\right)^{31}$.

The Borg Scale was explained, and participants were asked to maintain a mild (9) and slightly tiring intensity (13). ${ }^{4}$

The protocol lasted for 40 minutes, starting with 3 minutes of heating (power of $50 \mathrm{~W}$ ), increasing progressively the speed until reaching THR. For 35 minutes, the subject cycled at a rate that allowed maintenance in the defined THR interval. From the 38th minute until the 40th minute, the power and speed were gradually reduced to allow the heart rate to slowly lower, questioning the participant how he felt. ${ }^{31}$

\section{5 | Ethics}

The study was approved by the ethics committee (approval number: 1351/2018).

The participants were duly informed and clarified about the research project (rationale, procedures, and associated risks). In cases where there was no opposition from the participants, they signed an informed consent form, according to the Declaration of Helsinki.

In all phases of the study, the anonymity and confidentiality of the participants were ensured.

This study is applying for registration at ClinicalTrials.gov Identifier: NCT03594812.

\section{6 | Statistic}

Statistical Package for Social Sciences (SPSS) version 25 (IBM) was used for the analysis and statistical interpretation of the data, for a level of significance of $5 \%(P<0.05) .{ }^{32}$

The normality of the data was analyzed based on the ShapiroWilk test. ${ }^{32}$

Descriptive statistics were made with measures of central tendency (mean) and dispersion (standard deviation). ${ }^{32}$

To verify the homogeneity of the groups at the initial moment (M0), we used the $t$ test for independent samples. The $t$ test for paired samples was used to gauge the differences between moments. To compare the groups at the final moment, the difference variable in both groups (M1-M0) was calculated and the $t$ test for independent samples was applied. Given that the number of participants was equal in both groups, it was not necessary to apply the Levene test to verify the homogeneity of the variances. ${ }^{32}$

\section{7 | Results}

Based on the criteria defined and considering the volunteers who decided to join the study during the initial evaluation, a sample of 35 participants was created, being randomly distributed between the EG and PG. When contacted, only 28 participants agreed to advance their collaboration in the present study (Figure 1).

From the observation of Table 2 , it can be verified that the groups do not present significant differences among themselves in the characterization variables of the sample. The BMI values indicated that,
FIGURE 1 Sample constitution diagram

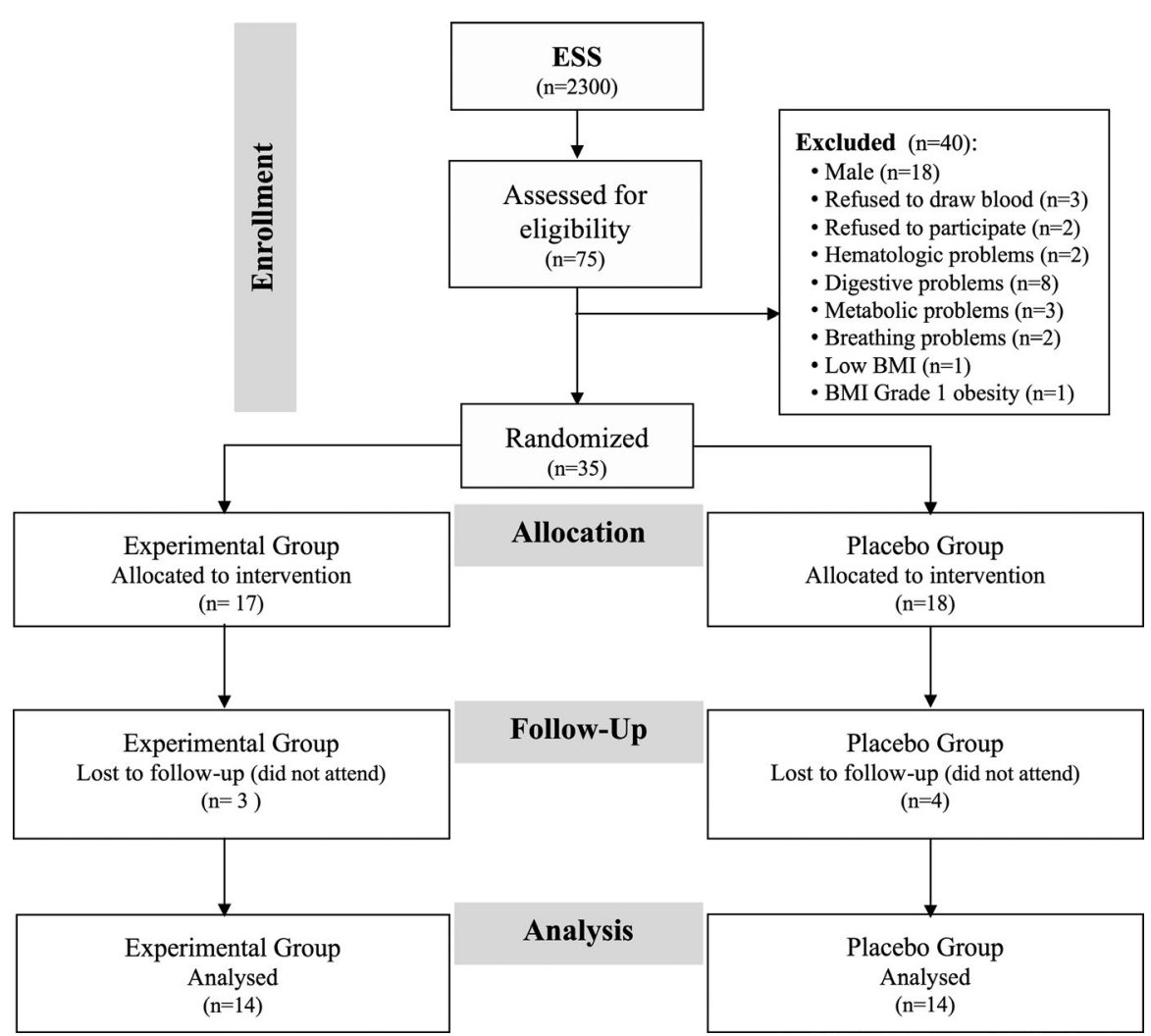


in both groups, the participants are mostly classified in the Normal category.

Regarding anthropometry and subcutaneous thickness values, no statistically significant differences were detected between the groups in $\mathrm{MO}(P>0.05)$.

The values of body mass, BMI, and total fat mass did not show statistically significant differences between the groups at the end of four sessions $(P=0.110, P=0.262, P=0.071$, respectively), but in the EG a statistically significant decrease was observed $(P=0.008$, $P=0.004, P=0.009$, respectively; Table 3$)$. The same did not occur in PG $(P=0.154, P=0.329, P=0.366$ respectively; Table 3$)$.

Regarding the waist circumference, waist/height ratio, subcutaneous adipose thickness, and horizontal abdominal fold, it was found that the EG presented a significant decrease in relation to $P G$ at the end of the four intervention sessions $(P<0.001, P<0.001$, $P=0.001, P<0.001$, respectively). From the initial to the final, in the $G E$ it was possible to detect a statistically significant decrease in the variables waist circumference, subcutaneous fat thickness and horizontal abdominal fold $(P<0.001, P=0.001, P<0.001$, respectively). In the PG, there were no significant differences in these variables ( $P=0.108, P=0.565, P=0.100$, respectively).

\section{3 | DISCUSSION}

After four combined sessions of aerobic exercise with RF, there was a statistically significant decrease in waist circumference, thickness of the subcutaneous adipose tissue, and horizontal abdominal fold between the two groups. Although lipolysis has not been proven to happen at the end of a session, it cannot be ruled out that there may be a summation effect of the sessions, culminating in the decrease in subcutaneous adipose tissue, as observed in the experimental group. The summation effect decreases tissue impedance by increasing blood flow. ${ }^{33}$ This decrease may justify a consequent decrease in adipose tissue.

The decrease in the horizontal abdominal fold may be further justified by the effects of RF at the level of the dermis. RF produces a stimulation of the activity of dermal fibroblasts which enhances the synthesis of collagen and a better organization of its fibers, leading to greater skin firmness. ${ }^{8}$ Although this is a common finding in articles of relevance in this subject, there is still no unanimous explanation for the mechanism of increase of skin firmness. ${ }^{3,8,12,13}$ Regarding the results found in the subcutaneous thickness difference variable, there were statistically significant differences between the groups, with a reduction in subcutaneous abdominal thickness in the EG. These results are consistent with the findings of this study and are supported by reports by Hayre et al, ${ }^{34}$ Mlosek et al, ${ }^{35}$ Suh et $\mathrm{al}^{36}$ and Wanitphakdeedecha et al ${ }^{37}$ justified by the increase of lipid substrate available to perform beta-oxidation during aerobic exercise.

In order to potentiate beta-oxidation, it was decided to maintain the exercise for 40 minutes at an intensity between $45 \%$ and $55 \%$ of the RHR, in order to guarantee the requirements for lipid oxidation in a global way. ${ }^{5}$

The training prescription guidelines for adipose mass loss recommend aerobic exercise, involving large muscle groups about five times a week. ${ }^{4}$ The intensity and duration of the exercise are preponderant factors to guarantee lipid oxidation. ${ }^{4}$ Body mass and BMI did not show significant differences between groups. This finding can be explained by the fact that both groups were subjected to an aerobic physical exercise protocol, with a number of sessions insufficient to generate significant adaptations at a global level, visible only in the long term and still determined by individual variability. ${ }^{38}$ At the same time, Boisnic et al, ${ }^{8} \mathrm{McDaniel}$ and Samková ${ }^{39}$ and Wanitphakdeedecha et $a^{37}$ affirm that the

\begin{tabular}{|c|c|c|c|c|}
\hline & \multicolumn{2}{|l|}{ Group } & \multicolumn{2}{|c|}{$\begin{array}{l}\text { Differences be- } \\
\text { tween groups }\end{array}$} \\
\hline & Experimental & Placebo & $t$ value & $P$ value \\
\hline \multicolumn{5}{|l|}{ IPAQ } \\
\hline MET'S (min/wk) & $2269.20 \pm 1160.80$ & $2157.97 \pm 1377.25$ & 0.239 & 0.813 \\
\hline \multicolumn{5}{|l|}{ QFA } \\
\hline Calories (kcal/d) & $2196.35 \pm 591.54$ & $2035.08 \pm 581.03$ & 0.753 & 0.458 \\
\hline Fat (g/d) & $94.85 \pm 47.40$ & $75.54 \pm 21.79$ & 1.431 & 0.163 \\
\hline Carbohydrates (g/d) & $239.71 \pm 69.21$ & $243.30 \pm 79.80$ & -0.132 & 0.896 \\
\hline Proteins (g/d) & $103.78 \pm 19.78$ & $101.39 \pm 28.85$ & 0.264 & 0.794 \\
\hline Age $(y)$ & $22.15 \pm 2.54$ & $21.00 \pm 1.71$ & 1.089 & 0.286 \\
\hline Body mass (kg) & $62.51 \pm 6.98$ & $58.38 \pm 5.62$ & 1.820 & 0.08 \\
\hline Height (m) & $1.65 \pm 0.07$ & $1.65 \pm 0.03$ & 0.844 & -0.199 \\
\hline $\mathrm{BMI}\left(\mathrm{kg} / \mathrm{cm}^{2}\right)$ & $23.00 \pm 2.55$ & $21.35 \pm 2.10$ & 1.840 & 0.078 \\
\hline
\end{tabular}

TABLE 2 Mean values and standard deviation of EG and PG for sample characterization: age, body mass, height, BMI, IPAQ and QFA

Note: Mean \pm Standard deviation.

Abbreviations: IPAQ, International Questionnaire on Physical Activity; MET'S, metabolic equivalent; QFA, Food Frequency Questionnaire; $t$ value, comparison between experimental group and placebo group with $t$ test for independent samples; value $P$, intergroup test value. 
TAB LE 3 EG and PG Anthropometry Values: Body Mass, BMI, Total Fat Mass, Waist Perimeter, Waist/Height Ratio, Subcutaneous Adipose Thickness, Horizontal Abdominal Fold

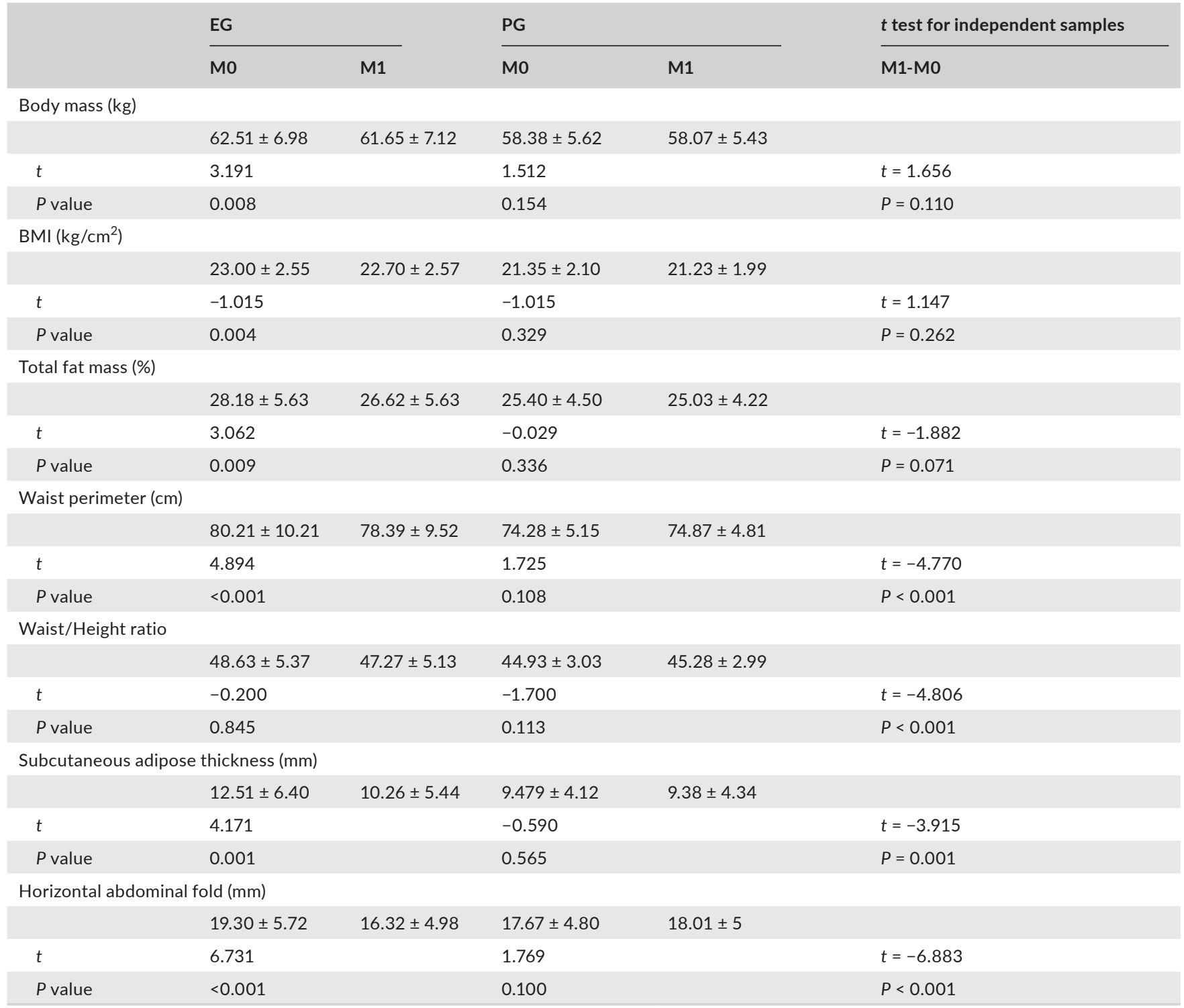

Note: Mean \pm Standard deviation; value $P$, intergroup test value; MO-M, difference variable $t$, test $t$ value.

decrease in body mass would not be an expected effect either by the isolated application of RF due to its exclusively localized effect. ${ }^{36}$ Although there were no differences between groups at the end, there was a statistically significant decrease in the EG from the initial moment to the final moment in the total fat mass and $\mathrm{BMI}$, indicating a higher tendency in this group to reduce body mass. The same did not happen in PG.

Although the waist-to-height ratio in the experimental and placebo groups did not suffer significant changes between $\mathrm{MO}$ and M1, this showed a variation in the opposite direction in each of the groups. Although the differences were not significant between the moments, the variation was sufficient to generate, at the end, statistically significant differences between groups, which is in agreement with the decrease of the waist circumference in the EG.
Anthropometric measures that measure central obesity, such as waist circumference and waist-to-height ratio, are better predictors of cardiovascular risk and metabolic complications than measures of total body fat such as BMI. ${ }^{40}$ Although the perimetry is not a parameter that allows to distinguish the type of mass evaluated, its evaluation was used to complement the other anthropometric procedures used, since the literature establishes a relation between the abdominal perimeter, waist/height ratio, visceral fat, and consequent cardiovascular risk. ${ }^{41}$ In the present study, there was a statistically significant decrease in waist circumference from the initial to the final moment in the EG, and this decrease was also significantly different from the PG, suggesting a possible influence of the intervention protocol on cardiovascular risk.

This study had as one of its main limitations the number of participants, since it was lower than necessary to make possible the 
generalization of the results. However, studies carried out on this

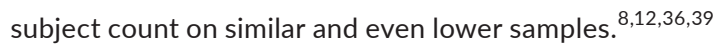

The other limitation of the study is that there was no objective control of presession dietary intake, and this question was minimized since the participants in both groups were in the same food conditions. The instrument used to monitor physical activity is limited in the specification of the practice of physical exercise and specific sports modalities. However, the groups were found to be homogeneous for this variable.

\section{4 | CONCLUSION}

After the four sessions, it was possible to observe that the intervention promoted a decrease in the waist circumference, the thickness of the subcutaneous adipose tissue and the abdominal fold in female subjects. Thus, the association of RF with physical exercise seems to be an adjunct to the reduction of abdominal adipose tissue. The intervention has shown to be an effective and safe tool to minimize cardiovascular risk by contributing to the decrease in waist/height and waist circumference ratio known cardiovascular risk factors.

\section{ACKNOWLEDGMENTS}

The research team would like to thank to BTL-Portugal and Doctor Leila Marques for providing the radiofrequency device and technical assistance for this study.

\section{ETHICAL APPROVAL}

School of Allied Health Technologies' Ethics Commission approval number: 1351/2018.

\section{REFERENCES}

1. World Health Organization. Health Observatory (GHO) Data Obesity. 2017. http://www.who.int/gho/ncd/risk_factors/obesi ty_text/en/. Accessed May 4, 2018.

2. Lopes C, Torres D, Oliveira A, et al. Inquérito Alimentar Nacional e da Atividade Física (2015-2016). Porto: U. Porto; 2017.

3. van der Lugt C, Romero C, Ancona D, Al-Zarouni M, Perera J, Trelles MA. A multicenter study of cellulite treatment with a variable emission radio frequency system. Dermatol Ther. 2009;22(1):74-84.

4. Pescatello L, Arena R, Riebe D, Thompson PD. ACSM's Guidelines for Exercise Testing and Prescription. Philadelphia: Wolters Kluwer/ Lippincott Williams \& Wilkins; 2014.

5. Jeppesen J, Kiens B. Regulation and limitations to fatty acid oxidation during exercise. J Physiol. 2012;590(5):1059-1068.

6. Mulholland RS, Paul MD, Chalfoun C. Noninvasive body contouring with radiofrequency, ultrasound, cryolipolysis, and low-level laser therapy. Clin Plast Surg. 2011;38(3):503-520, vii-iii.
7. Ahmadian M, Duncan RE, Sul HS. The skinny on fat: lipolysis and fatty acid utilization in adipocytes. Trends Endocrinol Metab. 2009;20(9):424-428.

8. Boisnic S, Branchet MC, Birnstiel O, Beilin G. Clinical and histopathological study of the TriPollar home-use device for body treatments. Eur J Dermatol. 2010;20(3):367-372.

9. Kaplan H, Gat A. Clinical and histopathological results following TriPollar radiofrequency skin treatments. J Cosmet Laser Ther. 2009;11(2):78-84.

10. Schulz KF, Altman DG, Moher D. CONSORT 2010 Statement: updated guidelines for reporting parallel group randomised trials. BMJ. 2010;340:c332-c332.

11. Roe E, Serra E, Guzman G, Sajoux I. Structural changes of subcutaneous tissue valued by ultrasonography in patients with cellulitis following treatment with the PnKCelulitis $\AA$ program. J Clin Aesthet Dermatol. 2018;11(3):20-25.

12. Goldberg DJ, Fazeli A, Berlin AL. Clinical, laboratory, and MRI analysis of cellulite treatment with a unipolar radiofrequency device. Dermatol Surg. 2008;34(2):204-209.

13. Emilia del Pino M, Rosado RH, Azuela A, et al. Effect of controlled volumetric tissue heating with radiofrequency on cellulite and the subcutaneous tissue of the buttocks and thighs. J Drugs Dermatol. 2006;5(8):714-722.

14. Pardini R, Matsudo $\mathrm{S}$, Araújo $\mathrm{T}$, et al. Validação do questionário internacional de nível de atividade física (IPAQ- Versão 6): Estudo piloto em adultos jovens brasileiros. 2001, R. Bras. Ci. e Mov. p. 45-51.

15. Craig CL, Marshall AL, Sjöström M, et al. International physical activity questionnaire: 12-country reliability and validity. Med Sci Sports Exerc. 2003;35(8):1381-1395.

16. Lopes C, Aro A, Azevedo A, Ramos E, Barros H. Intake and adipose tissue composition of fatty acids and risk of myocardial infarction in a male Portuguese community sample. J Am Diet Assoc. 2007;107(2):276-286.

17. Lintsi M, Kaarma H, Kull I. Comparison of hand-to-hand bioimpedance and anthropometry equations versus dual-energy X-ray absorptiometry for the assessment of body fat percentage in 17-18-year-old conscripts. Clin Physiol Funct Imaging. 2004;24(2):85-90.

18. Lopes I. Sagittal Abdominal Diameter: a Visceral Fat Indicator that Should be Taken Into Account in Clinical Practice. in Rev Esp Nutr Hum Diet. 2012:121-122.

19. Oliveira A, Nogueira T. Influência do Stretching Global Ativo na Flexibilidade da Cadeia Posterior e no Salto Vertical no Voleibol. in Rev Port de Fisio Desp. 2008:7-17.

20. Ribeiro-Filho FF, Faria AN, Azjen S, Zanella M-T, Ferreira S. Methods of estimation of visceral fat: advantages of ultrasonography. Obes Res. 2003;11(12):1488-1494.

21. Martins G. Sobre Confiabilidade e Validade. in Rev Bras Gest Neg. 2006:1-12.

22. Fan $M$, He P, Lyu J. Guidelines for Data Processing and Analysis of the International Physical Activity Questionnaire (IPAQ) - Short and Long Forms. 2005.

23. Gonçalves F, Mourão P. A Avaliação da Composição Corporal: A Medição de Pregas Adiposas como Técnica para a Avaliação da Composição Corporal. Motricidade. 2008;4(4):13-22.

24. Stolk RP, Wink O, Zelissen P, Meijer R, van Gils A, Grobbee DE. Validity and reproducibility of ultrasonography for the measurement of intra-abdominal adipose tissue. Int J Obes Relat Metab Disord. 2001;25(9):1346-1351.

25. Lauria AA, Santos TM, Amorim PRS, Marques FAD, Lima JRP. Predição da frequência cardíaca basal de indivíduos com níveis de atividade física altos e baixo. Rev Bras Med Esporte. 2013.

26. BTL, BTL - 6000 TR Therapy User's Manual. 2016.

27. FLIR, User's manual FLIR Ex Series. 2017, Intertek. 
28. Polar EO.Polar FT 7- User Manual. Polar Listen to your Body. Polar Listen to your Body. 2010.

29. Barbosa MP, da Silva NT, de Azevedo FM, Pastre CM, Vanderlei LC. Comparison of Polar(R) RS800G3 heart rate monitor with Polar(R) S810i and electrocardiogram to obtain the series of RR intervals and analysis of heart rate variability at rest. Clin Physiol Funct Imaging. 2016;36(2):112-117.

30. Chen MJ, Fan X, Moe ST. Criterion-related validity of the Borg ratings of perceived exertion scale in healthy individuals: a meta-analysis. J Sports Sci. 2002;20(11):873-899.

31. Wagganer JD, Pujol TJ, Langenfeld ME, et al. A workload selection procedure for the Astrand-Ryhming test for women. Percept Mot Skills. 2015;120(3):687-699.

32. Marôco J.Análise Estatística com o SPSS Statistics. 2014: Bertrand.

33. Trelles MA, Mordon SR. Adipocyte membrane lysis observed after cellulite treatment is performed with radiofrequency. Aesthetic Plast Surg. 2009;33(1):125-128.

34. Hayre N, Palm M, Jenkin P. A clinical evaluation of a next generation, non-invasive, selective radiofrequency, hands-free, bodyshaping device. J Drugs Dermatol. 2016;15(12):1557-1561.

35. Mlosek RK, Woźniak W, Malinowska S, Lewandowski M, Nowicki A. The effectiveness of anticellulite treatment using tripolar radiofrequency monitored by classic and high-frequency ultrasound. J Eur Acad Dermatol Venereol. 2012;26(6):696-703.

36. Suh DH, Kim CM, Lee SJ, Kim H, Yeom SK, Ryu HJ. Safety and efficacy of a non-contact radiofrequency device for body contouring in Asians. J Cosmet Laser Ther. 2017;19(2):89-92.

37. Wanitphakdeedecha R, Sathaworawong A, Manuskiatti W, Sadick NS. Efficacy of multipolar radiofrequency with pulsed magnetic field therapy for the treatment of abdominal cellulite. J Cosmet Laser Ther. 2017;19(4):205-209.

38. Egan B, Zierath JR. Exercise metabolism and the molecular regulation of skeletal muscle adaptation. Cell Metab. 2013;17(2):162-184.

39. McDaniel D, Samkova P. Evaluation of the safety and efficacy of a non-contact radiofrequency device for the improvement in contour and circumferential reduction of the inner and outer thigh. $J$ Drugs Dermatol. 2015;14(12):1422-1424.

40. Anunciação PC, Ribeiro R, Pereira MQ, Comunian M. Different measurements of waist circumference and sagittal abdominal diameter and their relationship with cardiometabolic risk factors in elderly men. J Hum Nutr Diet. 2014;27(2):162-167.

41. Gelber RP, Gaziano JM, Orav EJ, Manson JE, Buring JE, Kurth T. Measures of obesity and cardiovascular risk among men and women. J Am Coll Cardiol. 2008;52(8):605-615. 